\title{
Does a Recession have an Impact on Alcohol Consumption?
}

\author{
Johanna Dziadkiewicz, Alexia Pisani, Rebecca Wong
}

\begin{abstract}
The hypothesis of this paper is alcohol consumption will decrease when a country experiences a recession. We have used data covering the yearly consumption (in litres per capita) of alcohol from the World Health Organization (2015), combined with yearly \& quarterly GDP data from the OECD website (2015) to firstly identify years where there have been more than 2 periods of negative growth (recession), and to compare these data sets to see if any relationship exists. We have used correlation analysis between the financial data and the consumption data, as well as scatter graphs to see if there is a high correlation (0.7) or a trend, for 3 countries that have experienced a recession over the last 15 years (US, Finland \& Greece). What we found is that some types of alcohol did appear to show both positive and negative relationships to GDP or recession but this relationship differed between countries. There are other factors that must be considered including cultural relationship to alcohol in different locations, as well as any government or social programs.
\end{abstract}




\section{Introduction and hypothesis}

In 2014, the World Health Organisation (WHO) stated in their Global Alcohol Report that the most important factor affecting alcohol consumption is economic uncertainty (WHO 2014). Previous theoretical evidence suggests that there is a relationship between alcohol consumption and GDP, as well as a recession. One possible explanation for the dramatic change in consumption during a recession relates to whether the good is classified as inferior or normal. Increase in demand when GDP is increasing would lead to classification of alcohol as a normal good, or inferior if demand falls.

The hypothesis of this paper is: alcohol consumption will decrease when a country experiences a recession. The alternate hypothesis is: alcohol consumption will increase when a country experiences a recession.

To test our hypothesis we conducted a series of correlation analyses to look at the relationship of GDP and consumption of alcohol in periods with and without recession. The alternate hypothesis will be accepted if there is a strong positive correlation between a country's GDP in recession and alcohol consumption (above 0.7 ).

\section{Theory and literature review}

The theories of income effect and normal or inferior goods can give an explanation as to why consumption would change during periods of recession.

Income effect theory states that the quantity of a good a consumer demands changes with a change in income. For normal goods, less of the good is consumed when an income decreases. This theory would support the hypothesis that alcohol consumption will decrease when a country experiences recession. This is because during a recession household incomes will decrease as a result of the whole economy experiencing negative growth. 
Alternatively, if alcohol is considered an inferior good- increases in income would mean a reduction in consumption of that good, and conversely as income falls demand for the good increases.

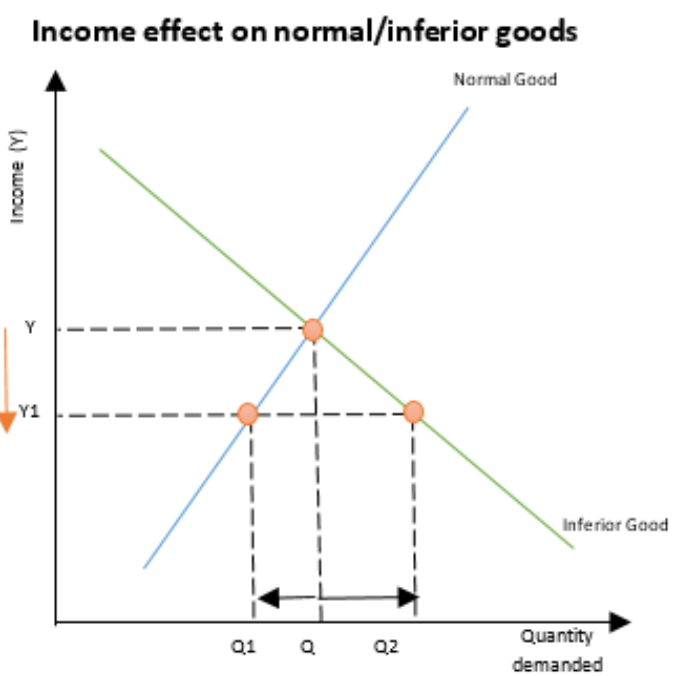

Figure 1: Income effect on normal/inferior goods

Both outcomes of the stated hypotheses in terms of the theory can be explained by the graph to the left. Assuming that alcohol is a normal good, we will see that as income decreases (recession), the quantity demanded would decrease to Q1. As for the alternate hypothesis that argues alcohol consumption increases when there is a recession, we are assuming alcohol is an inferior good and as such when income decreases quantity demanded increases to Q2.

There is a multitude of literature on the topic arguing both sides of the hypotheses. Proponents of the alternate hypothesis include Cotti, Dunn \& Tefft 2014 who concluded that macroeconomic indicators during a recession (unemployment rates and income per capita) were seen to impact on the demand for alcohol (Cotti, Dunn \& Tefft 2014). They found that demand for packaged alcohol and all major alcohol subtypes was positively correlated to unemployment levels, labelling it pro-cyclical.

The study by Mulia et. al (2014) analysed the 2009-2010 US National Alcohol Survey, in which they looked at the relationship of a recession-induced economic losses within households and alcohol usage. They found that severe economic loss- a person's job or housing- was positively correlated to positively correlate to alcohol consumption. Ruhm (2005) and Dee (2001) indicate that during periods of economic growth alcohol consumption has been statically proven to increase. This supports the alternate hypothesis from the perspective that a recession should have the alternate effect of decreasing during a recession. Studies argue that there is an increase 
in consumption levels (indicated by actual values and negative outcomes linked with alcohol consumption) in times of recession. With similar determinants of the outcome attempted by this study de Goeij et.al (2015) points to the income effect theory- concluding that people tend to spend less on normal goods during times of hardship due to budget constraints, however are more likely to self-medicate in psychologically stressful situations. This culminated in the finding that there seemed to be a rise in alcohol consumption during a recession.

In support of the null hypothesis a literature review found that the World Health Organisation were clear that when the factors resulting in a recession take hold on a country the recorded rates of consumption begins to decrease (WHO 2014). As GDP (income) decreases, people become less likely to purchase alcohol. This perspective is further bolstered by empirical studies by Evans and Moore (2012), who find a connection with the increase in surplus income in times of non-recession or growth, and the increased consumption of alcohol with income (Evans \& Moore 2102). This ties back to the idea that alcohol is a normal good and levels of consumption would fall in times of recession.

This empirical study by Ruhm (2007) shows trends over time where the economy are both in booms and troughs and indicates a strong trend to increasing consumption aligned with increased household income/positive economic growth. Indicating as per the theory that alcohol is a normal good.

This paper will contribute to these studies by specifically teasing out the direct relationship between GDP and alcohol consumption. Unlike other studies it will look at these two direct factors rather than relying on secondary data such as employment or income that are by products of GDP. Further this study will bring in to focus alcohol consumption and will be able to classify if the good is inferior or normal.

\section{Analysis}

To understand the change in alcohol consumption (litres per capita) this report analysed data from the WHO (World Health Organisation 2015) Data Repository by calculating the level of 
correlation with GDP (World Data Bank 2015), and whether the economy experienced a recession during that year. For the purposes of this study we have classified a recession as two periods of negative growth in GDP.

Scatter diagrams were also created to visualise this relationship and demonstrate the trend of each type of alcohol. Raw data can be found in the Appendix, for the purpose of this report periods of recession were coded with a 1 and periods without recession were coded with a zero. Key significant positive and negative changes are highlighted in green and red.

\section{1: United States of America}

In our analysis of alcohol consumption in the US, the report found that all types of alcohol had a low positive correlation with a state of recession- meaning that during a recession consumption tended to increase but at a low level.

However, all types of alcohol (as well as Wine and Spirits on their own) had a strong positive correlation (above 0.07) with GDP which could mean that they are normal goods. This is contradictory to the concept that sales increased during recession (and therefore fall when out of a recession). As GDP along with consumption will trend upwards over time, the results from the recession data are less likely to be a natural progression and as such more favourable of the alternate hypothesis.

Interestingly, Beer actually showed a strong negative correlation to GDP, showing that as GDP increased Beer consumption decreased and vice versa. This would indicate that Beer is an inferior good, moving in the opposite direction to GDP. However this is not evident in the correlation analysis to recession, where

Correlation of US consumption

\begin{tabular}{|c|c|c|c|}
\hline with $\operatorname{Rec}$ & & with GDP & \\
\hline US & recession & US & $g d p$ \\
\hline recession & 1 & gdp & 1 \\
\hline All types & 0.385742773 & All types & 0.952541012 \\
\hline Beer & -0.034138917 & Beer & -0.782125073 \\
\hline Wine & 0.22804452 & Wine & 0.973260879 \\
\hline Spirits & 0.280236268 & Spirits & 0.966930094 \\
\hline
\end{tabular}

Table 1: Correlation of US Alcohol consumption with Recession \& GDP 
beer consumption has a weak negative relationship, indicating that the cause of change could be attributed to other factors.

The average consumption of alcohol in the US is shown in the below line graph. The sections highlighted in grey are the periods of recession identified by this report. From the trend line it appears that average consumption for all alcohol types dropped. Thus indicating that when GDP drops alcohol can be deemed as a normal good as it follows the trend of the GDP.

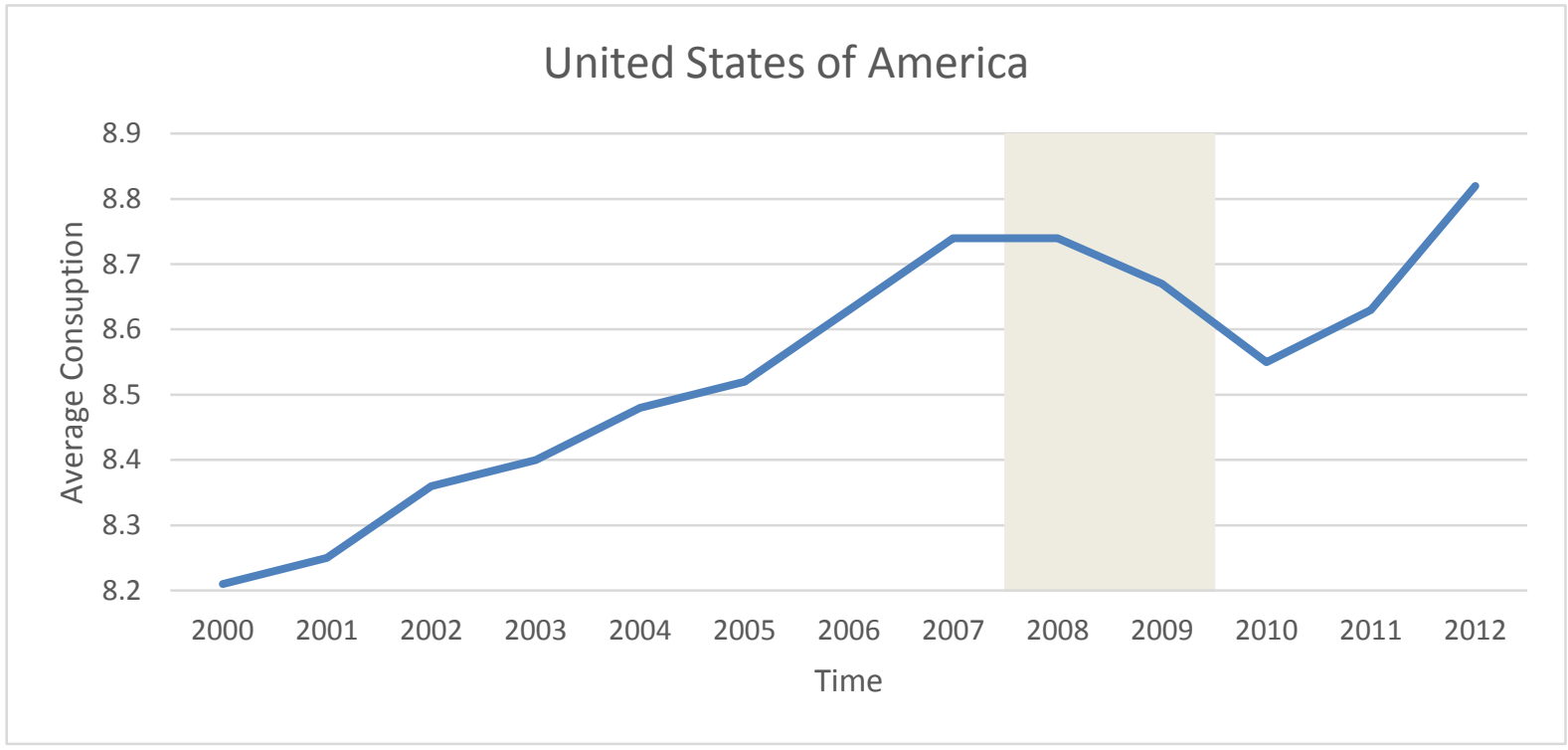

Figure 2: US Consumption Average 


\section{2: Finland}

Reviewing the correlation between alcohol consumption in Finland with recessions, there is a medium to high negative relationship here for Beer and Spirits, which would imply that sales in these two types of alcohol decrease during a recession (normal goods).

Beer has a similar level positive correlation to GDP which further backs up the concept that this is a normal good. This further supports the null hypothesis with the levels of mostly strong correlation between consumption and GDP in times of non-recession.

Specifically All types of alcohol and Wine have a high positive correlation

\section{Correlation of Finland consumption}

\begin{tabular}{lrlr}
\multicolumn{2}{l}{ with recession } & \multicolumn{2}{c}{ with recession } \\
\hline \multicolumn{1}{c}{ Finland } & \multicolumn{1}{c}{ Recession } & \multicolumn{1}{c}{ Finland } & \multicolumn{1}{c}{ Recession } \\
\hline Recession & 1 & Recession & 1 \\
All types & -0.380905808 & All types & -0.380905808 \\
Beer & -0.588365888 & Beer & -0.588365888 \\
Wine & 0.368278377 & Wine & 0.368278377 \\
Spirits & -0.560980896 & Spirits & -0.560980896 \\
\hline
\end{tabular}
to GDP showing that they are likely to increase with GDP (income) and that they are also normal goods. However, wine also has a low positive correlation to recessions, contradicting the theory that consumption falls with GDP in all cases however indicates that it is less positively correlated in times of recession than in times of normal GDP growth.

When plotting the average consumption we note that in the highlighted periods where the economy is experiencing a recession, average consumption of all products tends to decrease. 


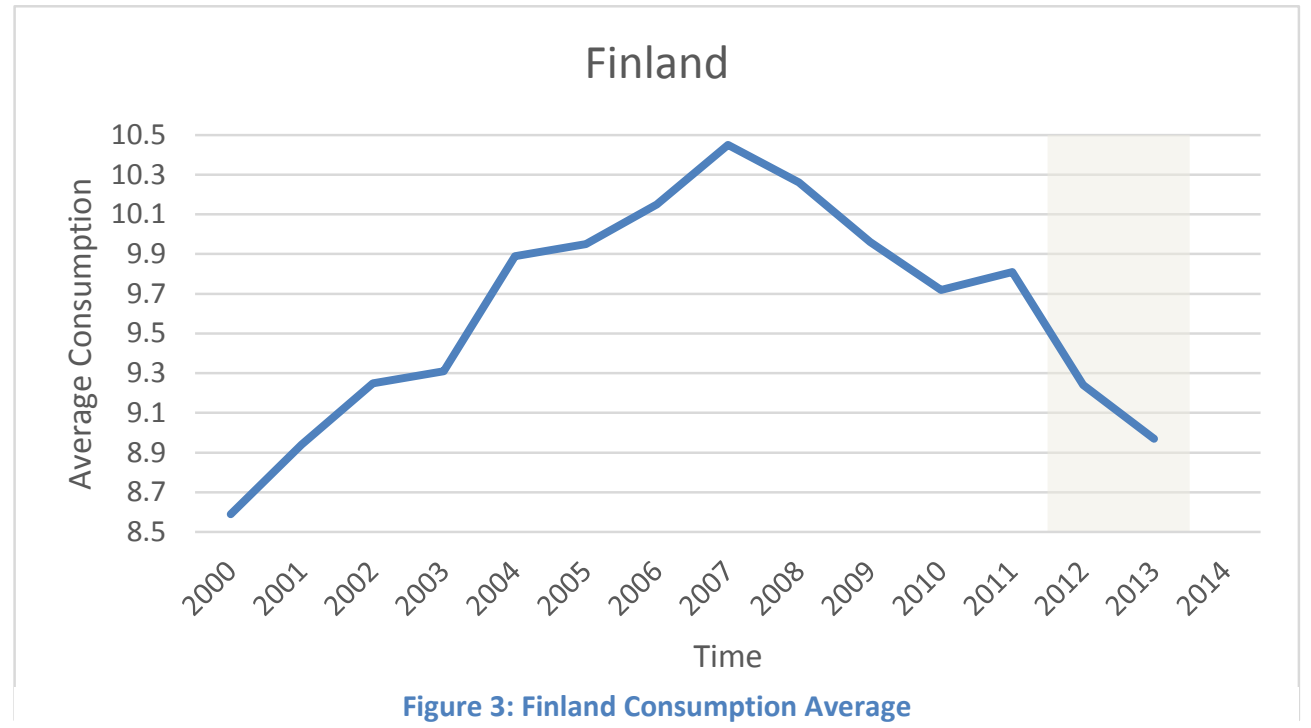

\section{3: Greece}

The analysis highlights that there is a high negative correlation between all types of alcohol \& spirits in recession. There is also a medium to high negative relationship between beer and recessions. This would indicate that alcohol in Greece generally is seen as a normal good, as consumption falls during a recession.

When compared with GDP the correlation results are much lower with all but wine showing a weak negative relationship. The negative relationship in particular with Spirits in recession allow us to reject the null hypothesis, as consumption is strongly

\section{Correlation of Greece consumption}

\begin{tabular}{|c|c|c|c|}
\hline ith Reces & & with GDP & \\
\hline & Recession & Greece & $G D P$ \\
\hline Recession & 1 & GDP & 1 \\
\hline All types & -0.757801328 & All types & -0.021659457 \\
\hline Beer & -0.604888489 & Beer & -0.217784631 \\
\hline Wine & -0.236560787 & Wine & 0.300437999 \\
\hline Spirits & -0.821532492 & Spirits & -0.260689329 \\
\hline
\end{tabular}


(above 0.7 ) negatively correlated to recession-indicating that consumption of spirits decreases during recession.

Average consumption for Greece dramatically decreases in the periods of recession highlighted in the below graph indicating that alcohol appears to be a normal good.

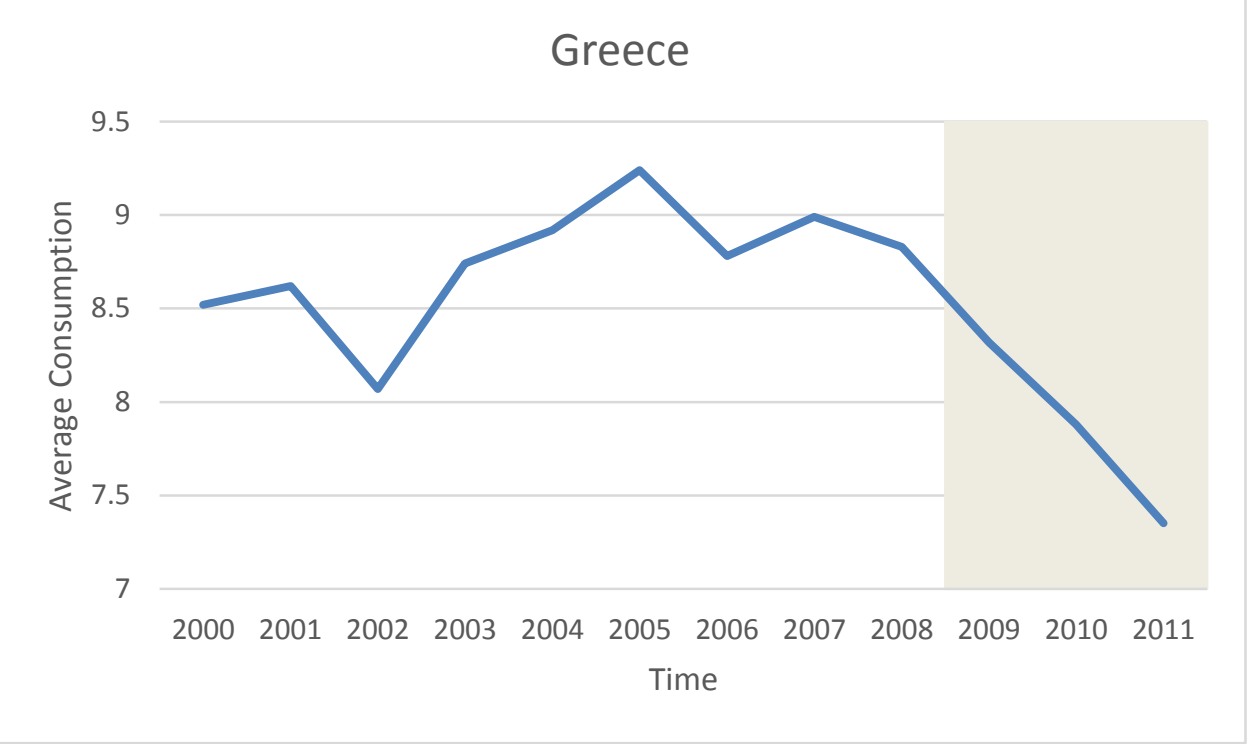

Figure 4: Greece Consumption Average 


\section{Conclusions and Limitations}

The data utilised in the analysis is restricted to three countries. The US and Finland both showed some relationship between economic performance and consumption of alcohol, but when comparing recessions to GDP the results were contradictory and may require further analysis to understand other factors that may be impacting our data. Of all three nations the US appears to be the most likely to increase per capita per consumption in recession followed by Greece. This variance between results could be accredited to differences in culture, and aspects of society which affect attitudes towards consumption of alcohol.

The WHO data on alcohol consumption is quite limited, and is only available from the year 2000. This meant that the number of records was limited to only 12-14 per country, and it is likely that there is not sufficient data to make an accurate conclusion.

In the report countries which have experienced a recession after the year 2000 were selected to ensure we can view the change in behaviour during or after a recession. The countries chosen are the United States of America (US), Finland \& Greece.

Other factors that might impact on our results include any social or government programs within these countries to reduce consumption of alcohol, or the possibility of substitutes that could create false or misleading data. Further the GDP per capita income varies substantially from nation to nation and hence may have a substantial impact on our findings.

Lastly we must consider the taxes on alcohol- in Greece the tax rate is relatively lower than in Finland, which may have a substantial impact and cause an increase in unrecorded rates of alcohol consumption in Finland as compared to Greece.

Overall the analysis in the previous section indicates that there are grounds to reject the alternate hypothesis, however this cannot be done with certainty. All three countries examined indicated that there was not a strong enough relationship (0.7) to deem the alternate hypothesis as accurate given that some of the data in recession indicate falling consumption. 


\section{References}

Bor, J, Basu, S, Coutts, A, McKee, M \& Stuckler, D 2013, 'Alcohol use during the great recession of 2008-2009.' Alcohol and alcoholism, vol. 48, no. 3, 343-348.

Cotti, CD, Dunn, RA \& Tefft, N 2014, 'The Great Recession and Consumer Demand for Alcohol: A Dynamic Panel-Data Analysis of US Households.' September 25, 2014, Available at SSRN 2184415.

Dee, TS 2001, 'Alcohol Abuse and Economic Conditions: Evidence from Repeated Cross-Sections of Individual-Level Data', Health Economics, vol. 10, no. 3, pp. 257-270.

De Goeij, MC, Suhrcke, M, Toffolutti, V, Van de Mheen, D, Schoenmakers, TM \& Kunst, AE 2015, 'How economic crises affect alcohol consumption and alcohol-related health problems: A realist systematic review.' Social Science \& Medicine, vol. 131, 131-146.

European Central Bank 2015, European Central Bank website, accessed 7/8/2015, https://www.ecb.europa.eu/home/html/index.en.html

Evans, WN, \& Moore, TJ 2012, 'liquidity, economic activity, and mortality', Review of Economics \& Statistics, vol. 94, no. 2, pp. 400-418.

Federal Reserve 2015, Board of Governors of the Federal Reserve website, accessed 7/8/2015, http://www.federalreserve.gov/

Harhay, MO, Bor, J, Basu, S, McKee, M, Mindell, JS, Shelton, NJ \& Stuckler, D 2014, 'Differential impact of the economic recession on alcohol use among white British adults, 2004-2010.' The European Journal of Public Health, vol. 24, no. 3, 410-415.

Haukeland, JV 1984, 'Sociocultural impacts of tourism in Scandinavia: Studies of three host communities', Tourism Management, vol. 5, no. 3, September 1984, Pages 207-214

International Monetary Fund 2015, International Monetary Fund website, accessed 8/8/2015, http://www.imf.org/external/index.htm 
Kahn, M 2015, 'Finland embarks on 'painful' austerity blitz', The Telegraph, http://www.telegraph.co.uk/finance/economics/11633816/Finland-embarks-on-painfulausterity-blitz.html

Luoto, R, Poikolainen, K, \& Uutela, A 1998, 'Unemployment, sociodemographic background and consumption of alcohol before and during the economic recession of the 1990s in Finland', International Journal Of Epidemiology, vol. 27, no. 4, pp. 623-629.

Mulia, N, Zemore, SE, Murphy, R, Liu, H \& Catalano, R 2014, 'Economic loss and alcohol consumption and problems during the 2008 to 2009 US recession.' Alcoholism: Clinical and Experimental Research, vol. 38, no.4, 1026-1034.

OECD 2015, Gross Domestic Product (GDP), OECD, accessed 8/8/2015, https://data.oecd.org/gdp/gross-domestic-product-gdp.htm

Ólafsdóttir, H 2015, 'Considerations on the Crash in Iceland and its effects on alcohol and drug issues.' Nordic Studies on Alcohol and Drugs, vol32, no. 3, 325-328.

Pacula, RL 2011, 'Substance use and recessions: What can be learned from economic analyses of alcohol?' International Journal of Drug Policy, vol. 22, no. 5, 326-334.

Ruhm, CJ 2005, 'Commentary: mortality increases during economic upturns', International Journal Of Epidemiology, vol. 34, no. 6, pp. 1206-1211.

The World Bank 2015, World DataBank website, accessed 8/8/2015, http://databank.worldbank.org/data/home.aspx

Ruhm, CJ 2007, 'A healthy economy can break your heart', Demography, vol. 44, no. 4, pp. 829-848.

World Bank Data 2015, World DataBank website, retrieved on 8 August 2015, http://databank.worldbank.org/data/home.aspx

World Health Organization 2014, Global status report on alcohol and health 2014, World Health Organization, retrieved 10 August 2015, http://www.who.int/substance abuse/publications/global alcohol report/en/ 
World Health Organisation 2015, Recorded alcohol per capita consumption 2000, World Health Organisation, retrieved 10 August 2015, http://www.who.int/en/

\section{Appendix: Data for Analysis}

\begin{tabular}{|r|r|r|r|r|r|r|}
\hline \multicolumn{7}{|c|}{ United States } \\
\hline Year & Recession & \multicolumn{1}{|c|}{$\begin{array}{l}\text { GDP } \\
\text { (Trilion }\end{array}$} & \multicolumn{1}{c|}{ All types } & Beer & Wine & \multicolumn{1}{c|}{ Spirits } \\
\hline 2000 & 0 & 11.55 & 8.21 & 4.62 & 1.17 & 2.42 \\
\hline 2001 & 0 & 11.67 & 8.25 & 4.66 & 1.17 & 2.42 \\
\hline 2002 & 0 & 11.87 & 8.36 & 4.66 & 1.25 & 2.46 \\
\hline 2003 & 0 & 12.21 & 8.4 & 4.58 & 1.29 & 2.54 \\
\hline 2004 & 0 & 12.67 & 8.48 & 4.58 & 1.32 & 2.57 \\
\hline 2005 & 0 & 13.09 & 8.52 & 4.5 & 1.36 & 2.65 \\
\hline 2006 & 0 & 13.44 & 8.63 & 4.54 & 1.4 & 2.69 \\
\hline 2007 & 0 & 13.68 & 8.74 & 4.54 & 1.44 & 2.76 \\
\hline 2008 & 1 & 13.64 & 8.74 & 4.54 & 1.44 & 2.76 \\
\hline 2009 & 1 & 13.26 & 8.67 & 4.43 & 1.44 & 2.8 \\
\hline 2010 & 0 & 13.60 & 8.55 & 4.28 & 1.48 & 2.8 \\
\hline 2011 & 0 & 13.82 & 8.63 & 4.24 & 1.51 & 2.88 \\
\hline 2012 & 0 & 14.14 & 8.82 & 4.28 & 1.59 & 2.95 \\
\hline
\end{tabular}

Table 4: United States Recession, GDP, Alcohol Consumption per capita (litres) 


\begin{tabular}{|r|r|r|r|r|r|r|}
\hline \multicolumn{7}{|c|}{ Finland } \\
\hline \multicolumn{7}{|c|}{ Consumption } \\
Year & Recession & \multicolumn{1}{|c|}{$\begin{array}{l}\text { GDP } \\
\text { (Trillion) }\end{array}$} & \multicolumn{1}{c|}{ All types } & Beer & Wine & Spirits \\
\hline 2000 & 0 & 1.80 & 8.59 & 4.27 & 1.07 & 2.22 \\
\hline 2001 & 0 & 1.85 & 8.94 & 4.37 & 1.19 & 2.32 \\
\hline 2002 & 0 & 1.88 & 9.25 & 4.43 & 1.31 & 2.37 \\
\hline 2003 & 0 & 1.91 & 9.31 & 4.37 & 1.43 & 2.38 \\
\hline 2004 & 0 & 1.99 & 9.89 & 4.57 & 1.42 & 2.79 \\
\hline 2005 & 0 & 2.04 & 9.95 & 4.59 & 1.47 & 2.82 \\
\hline 2006 & 0 & 2.13 & 10.15 & 4.57 & 1.55 & 2.86 \\
\hline 2007 & 0 & 2.24 & 10.45 & 4.7 & 1.64 & 2.86 \\
\hline 2008 & 0 & 2.25 & 10.26 & 4.62 & 1.67 & 2.69 \\
\hline 2009 & 0 & 2.07 & 9.96 & 4.54 & 1.68 & 2.49 \\
\hline 2010 & 0 & 2.13 & 9.72 & 4.47 & 1.7 & 2.33 \\
\hline 2011 & 0 & 2.18 & 9.81 & 4.61 & 1.74 & 2.25 \\
\hline 2012 & 1 & 2.15 & 9.24 & 4.27 & 1.7 & 2.16 \\
\hline 2013 & 1 & 2.12 & 8.97 & 4.28 & 1.71 & 2.03 \\
\hline
\end{tabular}

Table 5: Finland Recession, GDP, Alcohol Consumption per capita (litres) 


\begin{tabular}{|c|c|c|c|c|c|c|}
\hline \multicolumn{7}{|c|}{ Greece } \\
\hline \multicolumn{7}{|c|}{ Consumption } \\
\hline Year & Recession & GDP/capita & All types & Beer & Wine & Spirits \\
\hline 2000 & 0 & 19158.8213 & 8.52 & 2.4 & 3.69 & 2.38 \\
\hline 2001 & 0 & 20696.0886 & 8.62 & 2.35 & 3.78 & 2.44 \\
\hline 2002 & 0 & 22385.8901 & 8.07 & 2.37 & 3.14 & 2.5 \\
\hline 2003 & 0 & 23565.1639 & 8.74 & 2.24 & 3.9 & 2.54 \\
\hline 2004 & 0 & 25109.6576 & 8.92 & 2.21 & 4.18 & 2.48 \\
\hline 2005 & 0 & 25143.1457 & 9.24 & 2.2 & 4.52 & 2.47 \\
\hline 2006 & 0 & 28012.8652 & 8.78 & 2.25 & 4.03 & 2.46 \\
\hline 2007 & 0 & 29025.034 & 8.99 & 2.37 & 4.15 & 2.44 \\
\hline 2008 & 0 & 30872.385 & 8.83 & 2.39 & 4.01 & 2.4 \\
\hline 2009 & 1 & 30429.3097 & 8.32 & 2.25 & 3.73 & 2.31 \\
\hline 2010 & 1 & 28900.8593 & 7.88 & 2.22 & 3.73 & 1.91 \\
\hline 2011 & 1 & 26675.4024 & 7.35 & 1.99 & 3.81 & 1.55 \\
\hline
\end{tabular}

Table 6: Greece Recession, GDP, Alcohol Consumption per capita (litres) 